Article

\title{
Optimisation of Technological Processes by Solving Inverse Problem through Block-Wise-Transform-Reduction Method Using Open Architecture Sensor Platform
}

\author{
Konrad Kania ${ }^{1}$, Tomasz Rymarczyk ${ }^{2,3, *} \mathbb{1}$, Mariusz Mazurek ${ }^{4}$, Sylwia Skrzypek-Ahmed ${ }^{3}$, Mirosław Guzik ${ }^{5}$ \\ and Piotr Oleszczuk ${ }^{1}{ }^{\mathbb{D}}$ \\ 1 Faculty of Management, Lublin University of Technology Lublin, 20-618 Lublin, Poland; \\ k.kania@pollub.pl (K.K.); p.oleszczuk@pollub.pl (P.O.) \\ 2 Research \& Development Center Netrix S.A., 20-704 Lublin, Poland \\ 3 Faculty of Administration and Social Sciences, University of Economics and Innovation, \\ 20-209 Lublin, Poland; sylwia.skrzypek-ahmed@wsei.lublin.pl \\ 4 Institute of Philosophy and Sociology, Polish Academy of Science, 00-330 Warsaw, Poland; \\ mmazurek@ifispan.edu.pl \\ 5 Faculty of Transport and Computer Science, University of Economics and Innovation, 20-209 Lublin, Poland; \\ miroslaw.guzik@wsei.lublin.pl \\ * Correspondence: tomasz@rymarczyk.com
}

Citation: Kania, K.; Rymarczyk, T.; Mazurek, M.; Skrzypek-Ahmed, S.; Guzik, M.; Oleszczuk, P. Optimisation of Technological Processes by Solving Inverse Problem through Block-Wise-Transform-Reduction Method Using Open Architecture Sensor Platform. Energies 2021, 14, 8295. https://doi.org/10.3390/ en14248295

Academic Editors: Alessandro Floris and Roberto Girau

Received: 21 October 2021

Accepted: 7 December 2021

Published: 9 December 2021

Publisher's Note: MDPI stays neutral with regard to jurisdictional claims in published maps and institutional affiliations.

Copyright: (c) 2021 by the authors. Licensee MDPI, Basel, Switzerland. This article is an open access article distributed under the terms and conditions of the Creative Commons Attribution (CC BY) license (https:/ / creativecommons.org/licenses/by/ $4.0 /)$.

\begin{abstract}
This paper presents an open architecture for a sensor platform for the processing, collection, and image reconstruction from measurement data. This paper focuses on ultrasound tomography in block-wise-transform-reduction image reconstruction. The advantage of the presented solution, which is part of the project "Next-generation industrial tomography platform for process diagnostics and control", is the ability to analyze spatial data and process it quickly. The developed solution includes industrial tomography, big data, smart sensors, computational intelligence algorithms, and cloud computing. Along with the measurement platform, we validate the methods that incorporate image compression into the reconstruction process, speeding up computation and simplifying the regularisation of solving the inverse tomography problem. The algorithm is based on discrete transformation. This method uses compression on each block of the image separately. According to the experiments, this solution is much more efficient than deterministic methods. A feature of this method is that it can be directly incorporated into the compression process of the reconstructed image. Thus, the proposed solution allows tomographic sensor-based process control, multidimensional industrial process control, and big data analysis.
\end{abstract}

Keywords: big data; inverse problem; internet of things; cloud computing; quality of experience; optimisation; ultrasound tomography

\section{Introduction}

The paper's principal research goal is to present a solution based on the design of a proprietary Internet of Things device, data collection and storage mechanisms, and their analysis in energy optimisation of technological processes such as crystallisation, fermentation, two-phase flows, etc.

Advanced production process automation and control plays a crucial role in companies. They ensure high flexibility, rapid adaptation of manufacturing processes to evolving market demands, and safety and efficiency at optimum resources and energy costs. The development and implementation of advanced process monitoring mechanisms provide greater production flexibility. New technologies offer great implementation possibilities for industrial modernisation. They make the fourth industrial revolution possible. In the framework of Industry 4.0 (DFKI, Kaiserslautern, Germany), it is envisaged to use all types of intelligent devices supported by wired or wireless networks. Advances in the 
industrial Internet of Things and related fields such as industrial wireless networks (IWN), large data sets, and cloud computing help create a new concept of industrial environments based on constant monitoring processes. In particular, it is worth paying attention to the architecture of the prototype of the platform implementation, functions and features of each component layer, and the exchange of information between all types of devices. Cyber-physical systems are a new generation of digital systems, mainly focusing on the complex interdependence and integration between cyberspace and the physical world. A cyber-physical system consists of highly integrated computing, communication, control, and physical components. Related to the issue of a cyber-physical system is Industry 4.0 (DFKI, Kaiserslautern, Germany), which has created a revolution in manufacturing processes by interconnecting elements that allow different worlds such as physical, human, and digital to be linked together. Another of the terms to be noted is the Internet of Things and IoT services. Its conception to industry brings some concepts and technologies to develop industrial devices and device networks [1].

Industrial devices consist of sensors, actuators, and industrial robots connected through communication lines. Through this connection, monitoring analysis, data collection, and change are possible [2]. There are tremendous opportunities for change and competitive advantage in many industries using IoT. Real-time data are collected while the system is running to provide insights into system operations, which equipment is working well, and where there is downtime and why. Industry 4.0 (DFKI, Kaiserslautern, Germany) by itself, of course, does not have this information, and this information must be drawn from many interconnected elements. It combines standardisation, automation, smart devices, advanced diagnostics, and people into a common platform, which ultimately enables automation and digitisation to be able to solve problems [3].

The developed solution makes it possible to monitor and control the quality of production processes, analyze large data sets, and control through advanced interfaces. Current sensor technologies are primarily based on magnetic resonance and electrical tomography, including capacitive and resistive tomography. Electrical tomography measures voltage drops at the edge of the area to solve the inverse problem to allow image reconstruction by showing the corresponding conductivities. Ultrasound transmission tomography is more suitable for detecting connections between materials, whereas electrical capacitance tomography better characterises individual phases (liquid, solid, and gas). Electrical resistance tomography works well when visualising the concentration profile [4-9].

\subsection{Process Tomography}

The tomographic problem belongs to the inverse problem associated with some wave equations. The purpose of tomography is to analyze the properties of the object under study by performing measurements at its boundary [10]. Thus, tomography enables monitoring and a better understanding of industrial processes. Furthermore, tomographic imaging of objects allows the complexity of the structure to be analysed without interfering with the object.

Process tomography allows for analysing internal processes with no interference in the production process, identifying and diagnosing defects, flaws, and anomalies of various kinds. Reconstruction of images and individual elements of studied objects with tomography is possible even when we do not have access to their interior. More and more industrial processes require continuous monitoring and control. Furthermore, safety considerations require that such substances as oil, water, or gas be transported in an environmentally safe manner. It seems that new developments in the field of tomography can meet these demands. Thanks to developed, non-invasive techniques, it is possible to perform area studies of fluid flow, temperature distribution, or concentration of different substances without interfering with the technological process. In many cases, it is not possible to use other measuring techniques, such as optical tomography, which is not suitable for monitoring light-impermeable substances, or even X-ray tomography, in which the measurement time is so long that the observation of some processes is impossible. It 
seems then that impedance tomography, capable of spatial imaging of the distribution of electrical parameters of the studied medium, is the only solution [11].

The technique of process tomography is currently being attempted in various branches of industry, such as chemical, pharmaceutical, food, refinery, environmental protection, geology, and archaeology. Examples of practical applications include: measuring product homogeneity; visualisation of processes: mixing, separation, or polymerisation; liquid and gas flow control; monitoring of water bodies; rock and soil topography; groundwater contamination control; substrate investigations for the planning of gas pipelines drilling and directional drilling; examination of the condition of tree trunks; flame temperature testing; and detection of corrosion and damage in pipelines.

There is an overall tendency to grow the importance of predictive algorithms in industrial applications [12-18]. Industrial tomography is a non-destructive [19], non-invasive imaging technique applied to different industrial technologies to study physical and chemical processes without interfering with their interiors. The tomographic monitoring system continuously measures data, which allows for a better understanding and control of processes, enabling a quick response to any problems [20-22]. It simplifies process control in real-time and provides practical responses to system malfunctions. Industrial tomography may be turned into a powerful sensor for controlling dynamic processes. Distributed hardware infrastructure needs to perform various process discovery and startup tasks and is typically characterized by the spatial organization. Wireless sensor network (WSN) technology offers great opportunities for a wide range of devices to work together [23].

\subsection{Ultrasound Transmission Tomography}

The main purpose of tomographic studies is to non-invasively measure the interior properties of objects at their edges without interfering with their physicochemical characteristics [24]. Ultrasound waves are short waves and have properties of radiation and propagation. The wavelength depends on the medium under study, and the range varies from a few micrometres into tens of centimetres depending on the properties of the environment under study.

Moreover, using ultrasound, it is possible to perform multiple non-invasive measurements of the tested objects. The technology allows for measurement of the signal transit time, damping factor, and its derivatives after the frequency allow (with appropriate reconstruction transformations) for imaging the internal structure of the tested object. The image generated using appropriate reconstruction methods presents the internal structure of a given medium, resulting from measurements of scanning data at different angles after the ultrasonic pulses pass through the tested medium.

Ultrasound tomography, and in particular ultrasound computer tomography (UCT), can be broadly classified into two basic classes: diffraction and classical, and these in turn are divided into reflection and transmission [25-30].

\subsection{Architecture System}

The architecture of the presented solution consists of a cyber-physical system model, measurement sensors, and methods of analysis and classification of algorithms and image reconstruction. The system design was based on containers in the cloud computing model. The use of containers allows the use of public clouds. Using a distributed system using microservices and containers increases the system's flexibility in which individual modules perform clearly defined tasks. Failure of one of the modules does not cause the failure of the entire system. This architecture increases the level of reliability of the new IT system. It enables forecasting changes based on the analysis of historical data from the system and based on data obtained from devices in real-time.

Algorithms for manual and automatic control relate to issues related to data processing acquired from different probes placed at key nodes in the installation. The main feature of using wireless methods is obtaining essential information about the status of the process and installation by people of strategic importance in the management and technical 
supervision process. The transmitted data are analysed by an expert system and used to optimise production processes (Figures 1-3).

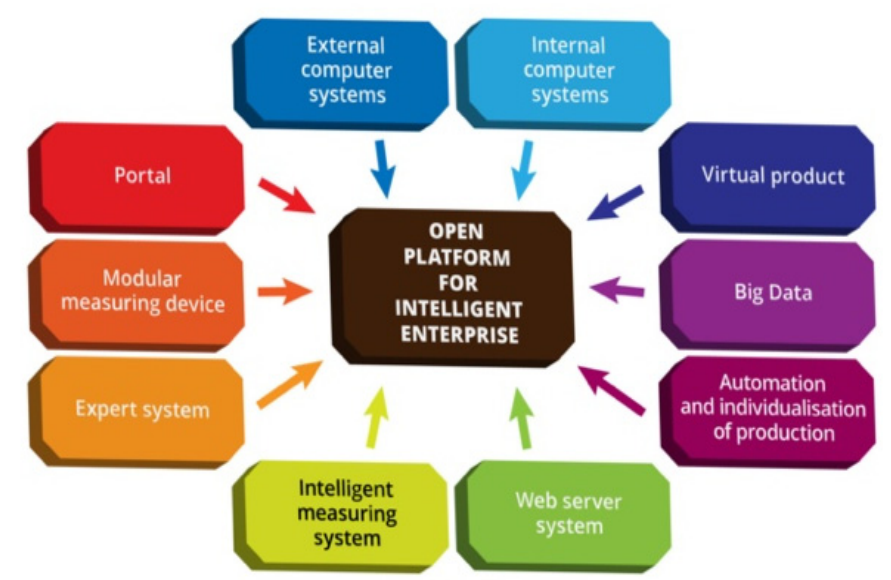

Figure 1. An open platform model for an intelligent enterprise system.

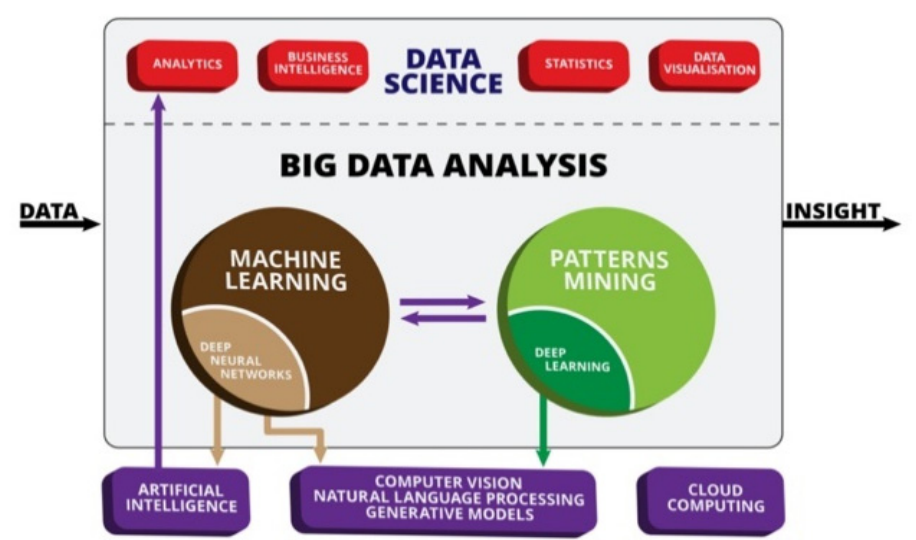

Figure 2. Analysis model of large data sets for machine learning.

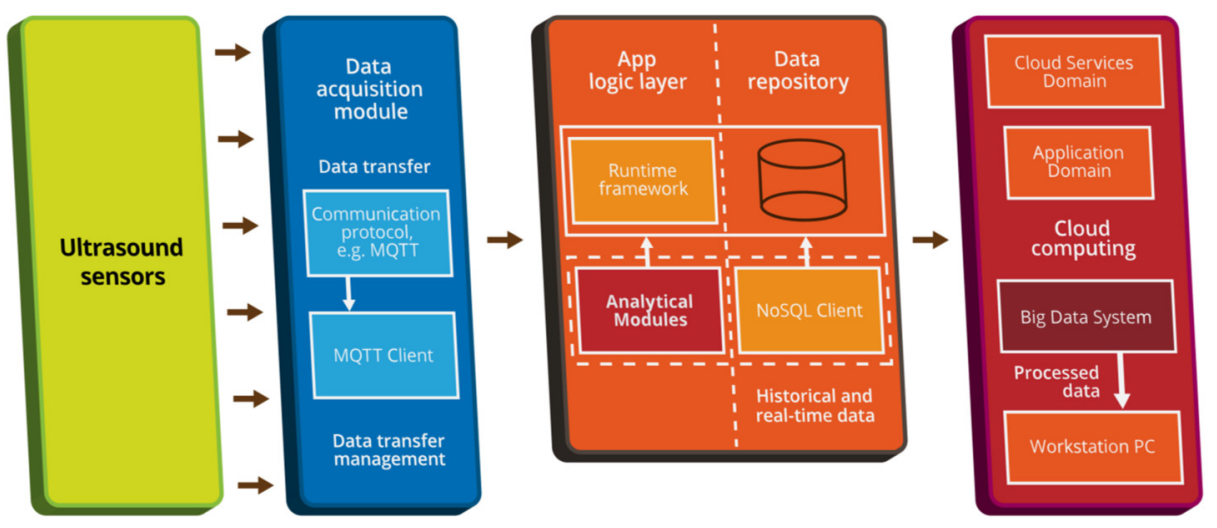

Figure 3. System structure model for cloud computing data processing, acquisition, collection, and analysis [22].

As part of the research, an expert system within the industrial tomography platform was designed for diagnostics and control of technological processes. To analyze the data collected during the technological process, based on their values and historical data, parameters are introduced to control the technological process, e.g., selecting the mixer's appropriate speed and adding substrates. The system enables mapping of the technological process in a digital twin, where the proposed settings are continuously verified and 
monitored. In addition, a streaming platform was created to transmit messages between microservices. The microservices include microservices responsible for image reconstruction, for technological process control, and for validation of user models, along with a database where all data received or sent are stored. The microservices include microservices responsible for image reconstruction, data interpretation, process control, and user model validation. The user can control the technological process using the microservice providing the user interface.

The IoT ecosystem includes systems at many levels. These include sensors and IoT devices, edge devices, cloud computing systems, mobile applications, network interfaces, and software. The tasks facing IoT security systems are very extensive. These include, for example, avoiding situations where the acquisition of a single device can lead to the acquisition of an entire device collection.

However, if such a situation occurs, it is necessary to prevent subsequent attacks by the devices taken over (particularly Distributed Denial of Service (DDoS) attacks). Threats may cause irregularities to the following elements of the IoT system infrastructure: insufficient security level of Internet interfaces for IoT platforms; the low-security level of IoT devices and network interfaces misinterpreted security of protocols and communication channels; low-security level of ecosystem services cloud computing; low-security level of applications running in the cloud, on mobile platforms, and IoT devices; incorrectly functioning IoT system interface security (API) systems; erroneously designed ways to secure data transfer over the network; and insufficient security level of transport layer security.

Transport layer security (TLS) ensures secure data transmission over the network. The data are encrypted to prevent eavesdropping and analysing the content. TLS (also known as SSL) is widely used to provide secure access to, for example, websites. TLS ensures a trusted connection between the server and client before transferring data. It does this by using server certificates that clients must verify. In some cases, the server also checks client-specific certificates.

The MQ Telemetry Transport (MQTT) data transfer protocol uses TCP as the transport protocol, but by default, the connection between the device and the broker and the broker and the downstream client does not use encrypted communication. The implementation of TLS affects data transfer performance and server load; however, for security reasons, most MQTT brokers support the use of TLS to allow applications to exchange confidential data securely. It is assumed that port 8883 is used for secure communication, and the normalised name is accepted as "secure-MQTT". However, security is associated with greater CPU utilisation and increased communication costs. Although the additional use of processor resources is usually irrelevant to the broker, this can be a problem for devices with low computing performance that are not designed for tasks that require increased computing power.

WebSocket is a communication protocol that ensures a full-duplex connection over a single TCP connection. In addition, WebSocket (Computer Communications Protocol) allows sending message streams via TCP. WebSocket is different from the HTTP protocol, but it is compatible with it to communicate through ports 80 and 443. Similarly, for secure communication, TLS and port 443 will be used. Furthermore, WSS (WebSockets over SSL/TLS) is encrypted, which protects against man-in-the-middle attacks. Unfortunately, WebSocket does not support authorisation or authentication. One of the schemes that seemed to solve the WebSocket authentication problem is an authentication system based on encrypted strings called "tickets". The accepted authentication standard for WebSocket and Rest-Ful API is JSON Web Token (JWT).

In its compact form, JSON Web Token consists of three parts separated by dots (.): header, load, and signature. When a user logs on using his credentials during authentication, the JSON network token will be returned. Because tokens are credentials, special care should be taken to prevent security problems. The operator agent needs to send a JWT, usually in the authorisation message header, by the Bearer scheme every time a user requests access to a secured route or resource. In some cases, this may be a stateless 
authentication mechanism. Resources protected by the server will require validation of the header. The whole process can be presented in the following steps: (a) The client or application asks for authorisation from the authorisation server. It is done by one of several authorisation methods (e.g., OpenID Connect); (b) Once authorisation is granted, the authorisation server returns an access token to the application; (c) The application then uses the access token to access a protected resource (e.g., Restful API).

The concept of the Internet of Things assumes intelligent communication and analysis of the collected data, which in response is to ensure the improvement of decision-making processes. An adequately designed technological line is designed to transfer the read results between devices. These data are processed and analyzed in an appropriate IT system that communicates and integrates individual elements of an intelligent production line. However, this communication is not limited only to the devices operating in the enterprise but goes beyond the internal infrastructure, e.g., by analyzing data received from external suppliers to optimize production or logistics processes. Production lines consist of sequences of stations or machines that are used for serial and mass production when combined. The designed model of optimization and pre-processing enables for process optimization. This approach allows to create customized analyses and optimizations by automating system operation using measurement and control data.

The measurement system can collect data from different variants of object distribution and anomalies as historical data that can be used in deep learning processes. Two variants are possible here, the first by collecting data from the numerical model with appropriate noise and using them later in the training process. The second option is collecting real data from the measurement system, which can also be used in the machine learning process.

The designed solution will serve to improve the production processes of enterprises using technological processes consisting in mixing liquid or lose ingredients and using physical or chemical reactions at the stage of the technological process. According to the available data on the market, there are developed systems for monitoring the technological processes of production enterprises. However, these solutions use methods other than tomography, which is associated with lower measurement accuracy. Furthermore, the methods used limit the functionality and possibilities of the existing systems. Our system is the only complimentary solution based on a holistic approach to the processing system. The system will make it possible to check whether the processes related to the production of beverages, food, cosmetics, and medicines are carried out correctly in industrial installations and will optimize these processes. It will non-invasively examine the processes in closed flows in a complex system of pipes and reactors. These will be processes related to, inter alia, dairy products, beverages, cosmetics, and medicines. The system will enable monitoring of mixing, crystallization, filtration, separation processes, level detection on dispersed surfaces, multiphase fluids of oil, water and gases, and pneumatic transport analysis. The system is especially useful when small changes to the recipe lead to significant changes in the physical properties of the product components, and it allows the determination of changes in concentration over time. Studying the suspension deposition process and flavour extraction is important for food, cosmetic, and drug manufacturers. The information provided by the system will be used to select different mixing options, optimize performance, and measure product quality. The validation was carried out in laboratory conditions. For this purpose, a measuring stand was prepared as a tank (reactor), and various types of phantoms and technological processes were tested. The research aimed to analyze the quality of the reconstruction of the properties of the studied environment in order to be able to make an optimization decision in an expert system on this basis.

\subsection{Structure of the Paper}

This article is organised as follows: Section 1 shows state of the art regarding tomographic algorithms used to reconstruct images, emphasising the Industry 4.0 concept and the Internet of Things. A description of the research and novelty contributions included 
in the concept presented is given in Section 2. This section includes a characterisation of the test rig as well as the algorithm used. Section 3 shows some example reconstructions achieved by the BWTR method based on accurate measurements. An evaluation of the quality of the reconstructions obtained by the BWTR method is also performed. Three metrics were applied as image reconstruction quality measures: mean square error (MSE), image correlation coefficient (ICC), and relative image error (RIE). Finally, Section 4 summarizes the highlights of the research work, the results developed, and the conclusions drawn. Plans for future research are also presented.

\section{Materials and Methods}

\subsection{Measuring System}

The ultrasonic tomograph is entirely responsible for measuring sequence management, setting up active probes, and capturing and collecting data from the probes. It is additionally equipped with a USB A port for saving results on a portable drive; a USB B 2.0 port for controlling and transferring results; and an Ethernet port for transferring results via TCP and UDP protocols. Figure 4 shows an active measuring transducer. The active transducer acts as both receiver and transmitter of the ultrasonic signal.

The control of the probe and the readout of its measurements is done via the CAN 2.0A bus. (Figure 4 ).

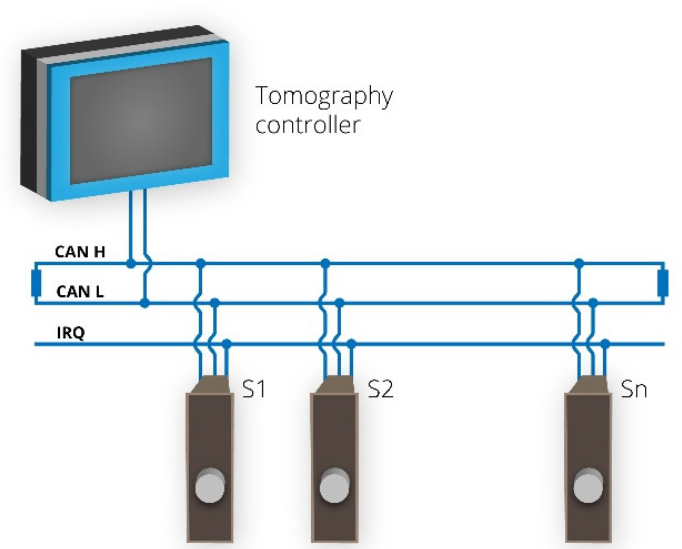

(a)

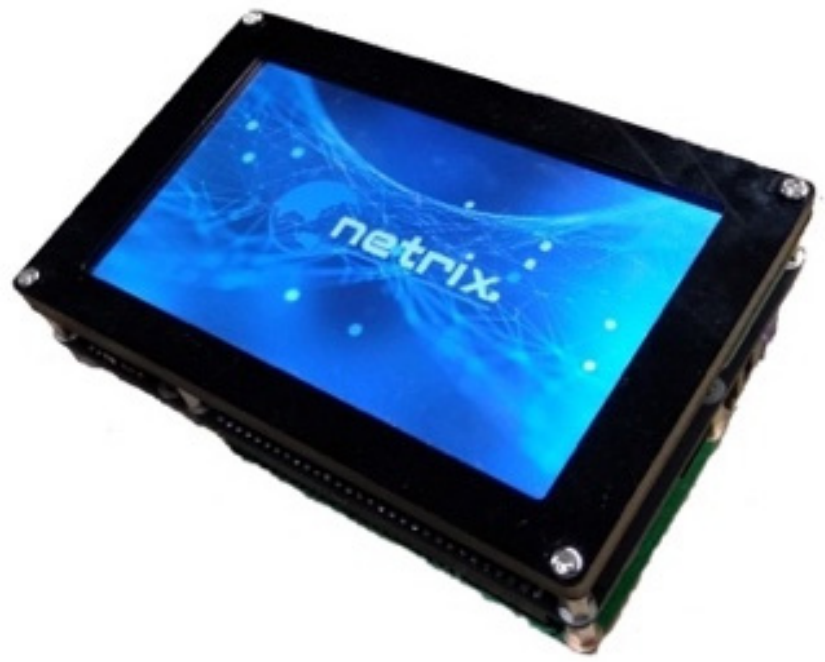

(b)

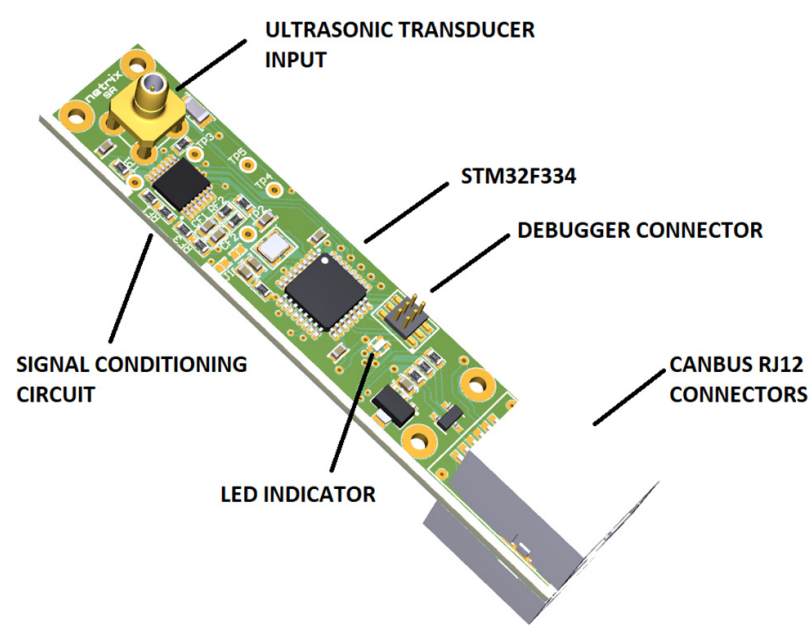

(c)

Figure 4. Ultrasound tomography device: (a) block diagram, (b) start-up control module, (c) active measuring transducer. 
The measuring setup presented includes a dedicated measuring architecture, including transducers (Figure 5).

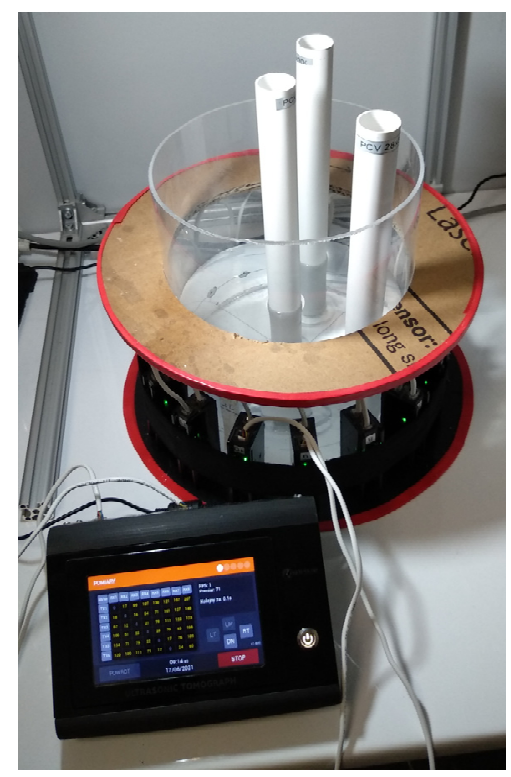

Figure 5. Measurement system for ultrasound tomography.

The sensors are constructed in such a way that they can be positioned very closely together. The interface of the controller was implemented in English and Polish (Figure 6).

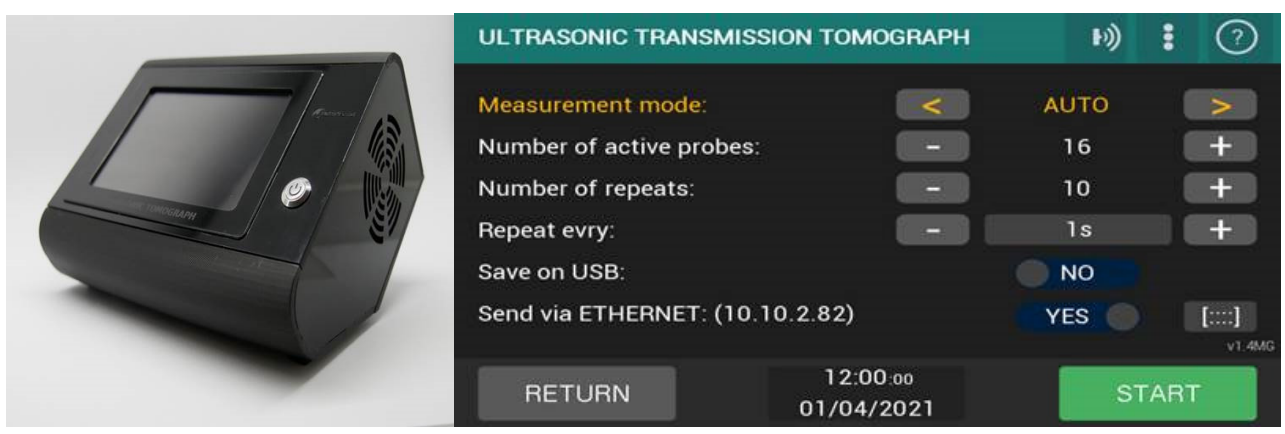

Figure 6. The main module of the UST measurement system and view of the configuration screen.

\subsection{Block-Wise-Transform-Reduction Method Based Image Reconstruction}

A major problem with non-invasive tomographic methods is the small number of measurements relative to the pixels reconstructed. The image reconstruction problem often leads to a redundant system of algebraic equations. The radical matrices appearing in the equations are numerically very sparse, including the consolidation of the inverse operation range [31-34]. Instead of reconstructing the whole image inaccurately pixel by pixel, by using lossy image compression, the number of unknowns is reduced, which is the main feature of the presented method.

As the name of the method suggests, the compression involves partitioning the picture into square-shaped blocks and using a transformation (which enables compression) to apply to each block individually. The discrete cosine transform (DCT) was applied for such compression, and $48 \times 48$ pixel resolution tomograms were divided into $8 \times 8$ pixel blocks as a starting point, giving 36 blocks distributed on a 6 by 6 grid. It was verified that the application of the block transform enables correct compression and decompression of tomograms, which provided a basis for testing on real measurement data. The tests performed showed that the application in tomographic imaging is feasible and can produce satisfactory results. In order to test the effect of image compression on the overall quality 
of reconstructed images typical for industrial tomography applications, we can create an example synthetic image containing three oval inclusions, each with a different value in the reference image. We subject the master image to a lossy compression process and verify that the basic image properties are preserved after decompression.

The results of the test are shown in Figure 7.

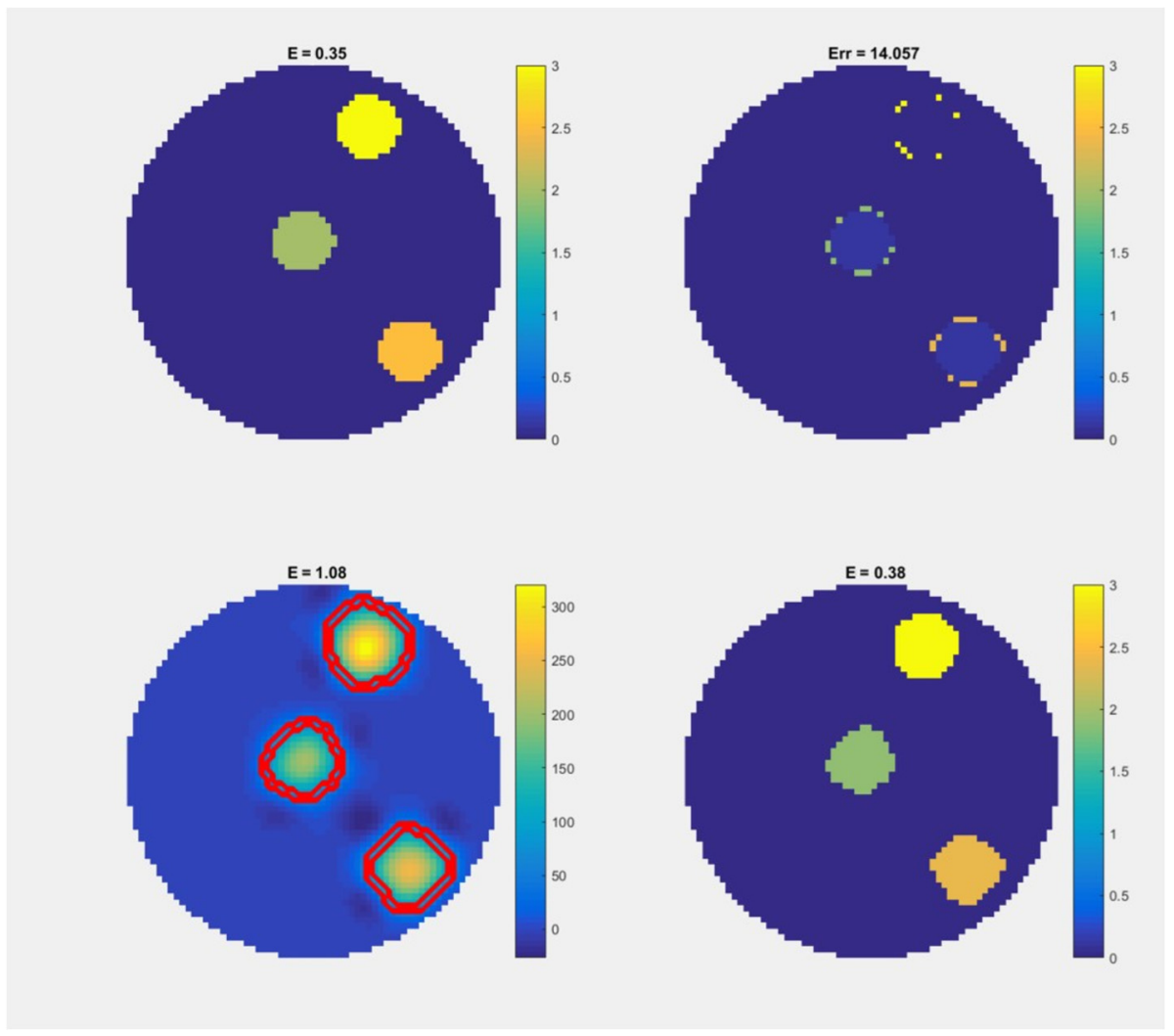

Figure 7. Examples of direct image decompression with filtering for three phantoms.

The upper left corner shows the master image. The lower-left corner shows the decompressed image that was additionally segmented (red lines in the image). The image in the bottom right corner shows the desegmented areas-the colour of the desegmented area results from averaging the values in the decompressed image. Finally, the image in the upper right corner is different between the desegmented areas and the reference image.

The test shows that despite the use of lossy compression, the reconstructed image retains its most salient features: position, size, and shape of the inclusions, and it is possible to reconstruct the original values of each inclusion. Further tests and analyses of the effect of compression on the quality of the reconstructed image are presented later in this paper.

\subsubsection{Discrete Cosine Transform (DCT)}

A fragment of an image $(8 \times 8$ pixels $)$ of the picture can be treated as an $8 \times 8$ matrix, which can be thought of as a linear combination of matrices from the canonical basis, that is, a set of matrices filled with zeros except for one element equal to one (Figure 8). The visualisation of all 64 base vectors of DCT for $8 \times 8$ pixels images is shown in Figure 9 . 


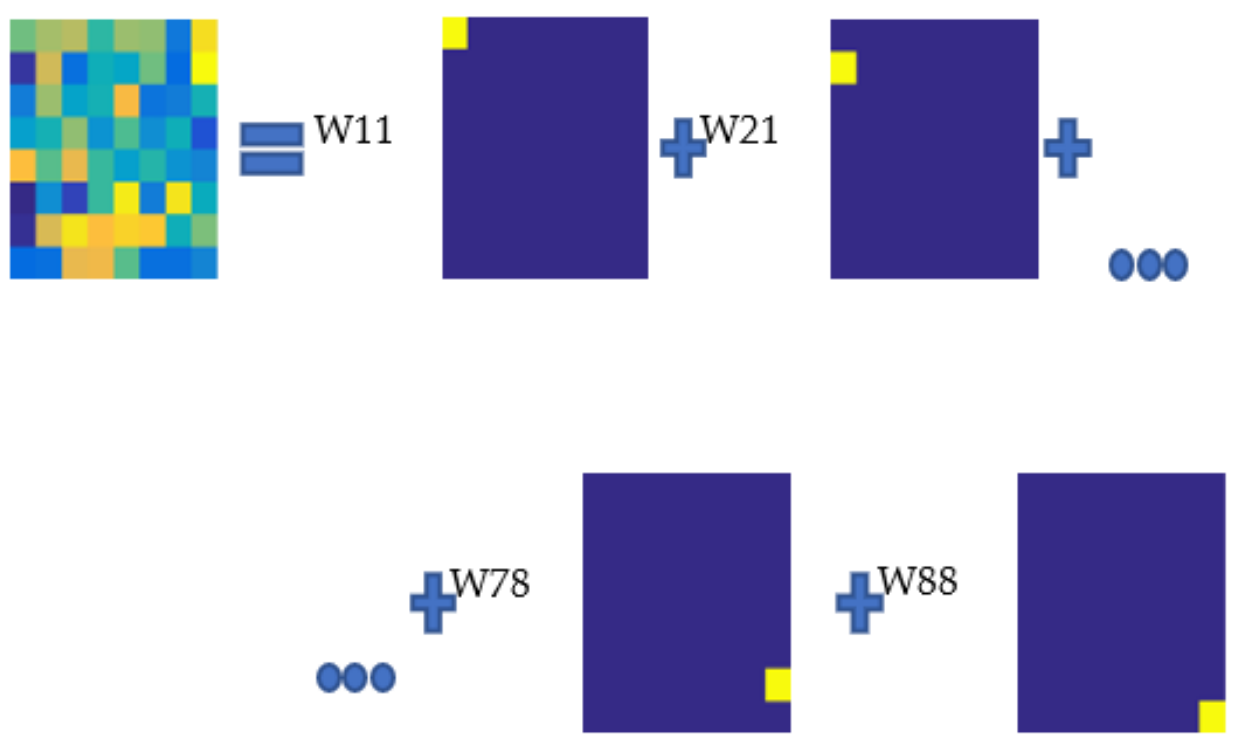

Figure 8. Visualisation of algebraic interpretation of the uncompressed image.

Mathematically:

$$
I_{m}=\sum_{p, q=1}^{8,8} w_{p q} F_{p q}
$$

where $I_{m}$ is an image block, $w_{i j}$ are numerical factors, and $F_{p q}$ are base images.

The discrete cosine transform involves changing the canonical basis to an orthonormal one, which encodes the image content at increasingly higher frequencies instead of encoding individual pixel information.

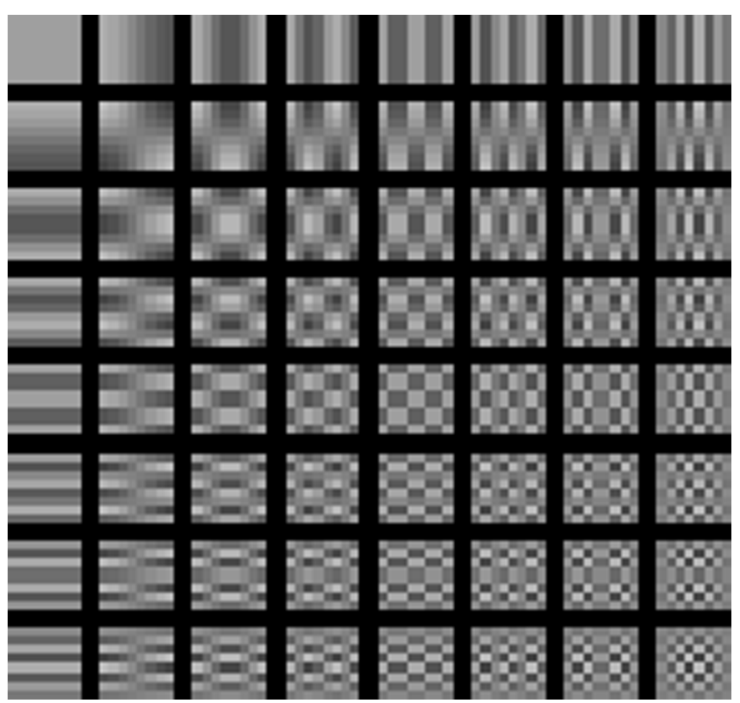

Figure 9. Visualisation of all 64 base vectors of DCT for $8 \times 8$ pixels images.

Equations describe base images $F_{p q}$ in DCT:

$$
F_{p q}=\alpha_{p} \alpha_{q} \cos \frac{\pi(2 m+1) p}{2 M} \cos \frac{\pi(2 n+1) q}{2 N}
$$

where:

$$
\alpha_{p}=\left\{\begin{array}{l}
1 / \sqrt{M} \\
\sqrt{2 / M}
\end{array}\right.
$$




$$
\left.\begin{array}{c}
p=0 \\
1 \leq p \leq M-1
\end{array}\right) \begin{gathered}
\alpha_{q}=\left\{\begin{array}{l}
1 / \sqrt{N} \\
\sqrt{2 / N}
\end{array}\right. \\
q=0 \\
1 \leq q \leq N-1
\end{gathered}
$$

Equations can describe the matrix of coefficients in the DCT base:

$$
B_{p q}=\alpha_{p} \alpha_{q} \sum_{m=0}^{M-1} \sum_{n=0}^{N-1} \mathrm{~A}_{\mathrm{mn}} \cos \frac{\pi(2 m+1) p}{2 M} \cos \frac{\pi(2 n+1) q}{2 N}
$$

The concept of discrete cosine transform is to change the fundamental matrix $F_{p q}$ into another base of non-dependent pictures with the feature that the successive base functions bring less information with high frequencies. By getting the information out of a particular pixel, the image degrades significantly as we lose information about the pixel's value. By moving to frequency decomposition, we can eliminate the high frequencies (without affecting image quality).

\subsubsection{Compression Tests}

We used a tomographic measurement system that contained 16 measurement probes, from which one can obtain 120 measurements per one frame. Using the first 3 (of all 64) coefficients of the transform, we have $108(3 \times 36)$ coefficients to determine, and thus, the problem ceases to be overdetermined.

Using only 3 of 64 coefficients makes such compression seem to be quite drastic, raising the question of whether we can get any satisfactory image with such a severe compression. Because of that, several synthetic tests were conducted. First, it was verified what shapes could be obtained with only three base functions. The convex envelope of these functions is then visualised in Figure 10 according to the following definition (allowing for meaningful visualisation):

$$
\operatorname{span}_{2 d}\left\{F_{11}, F_{12}, F_{21}\right\}=\left\{w_{11} F_{11}+w_{12} F_{12}+w_{21} F_{21}: w_{11}=0, \mathrm{w}_{12} \in[-1,1], \mathrm{w}_{21} \in[-1,1]\right\}
$$

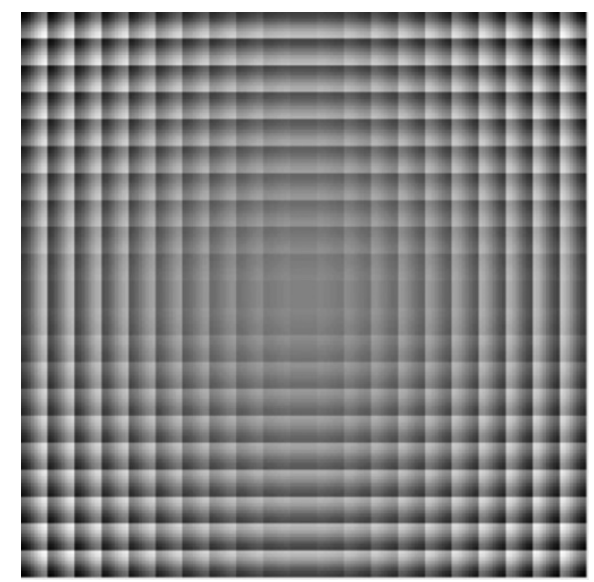

Figure 10. Visualisation of the convex envelope of $\left\{F_{11}, F_{12}, F_{21}\right\}$ defined in (4).

Notice that despite only three components, we should reconstruct the oval shapes in the image.

A synthetic image of very good quality can be seen as the two circular inclusions in Figure 11 on the left. In contrast, on the right is a compressed image of the left one (up to 3 factors for each $8 \times 8$ block of pixels) with no additional processing. The image left 
much to be desired, but the location of the bores is approximately retained. Values over the images are the entropy computed for the picture.

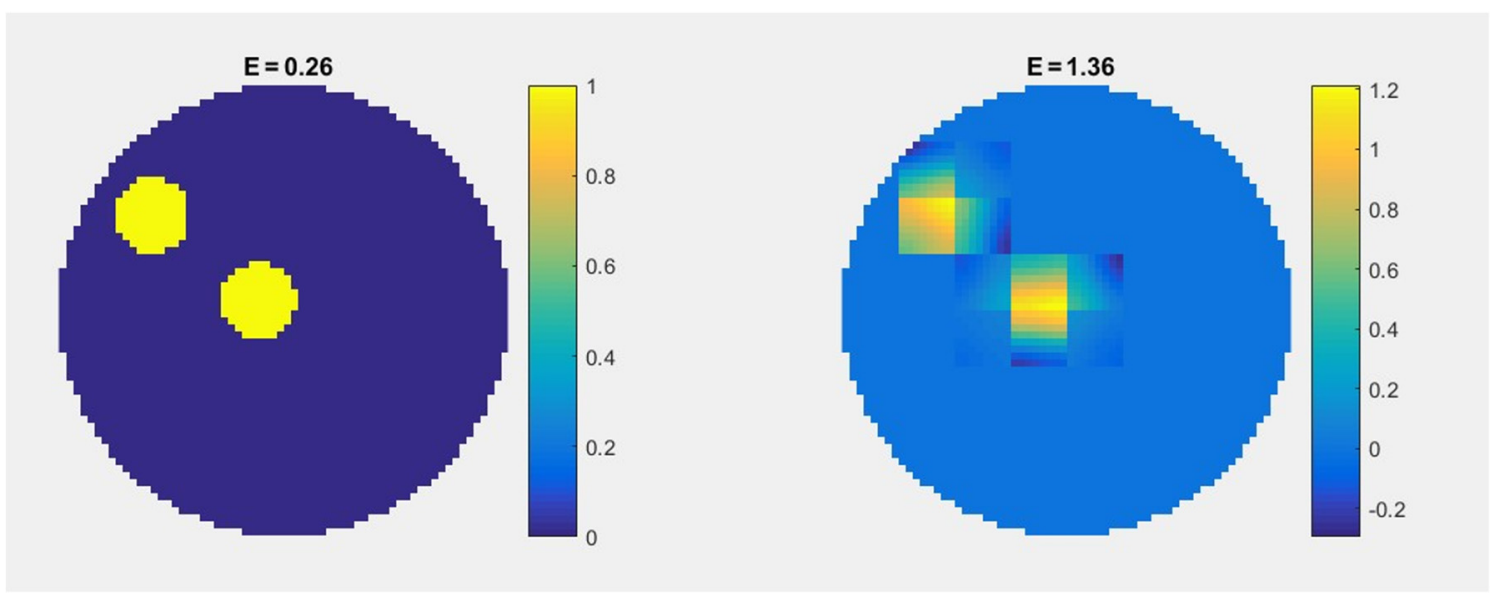

Figure 11. Direct decompression.

After direct decompression, the image can be further processed by filtering with a static high-pass filter and further segmentation, as seen in Figure 12.

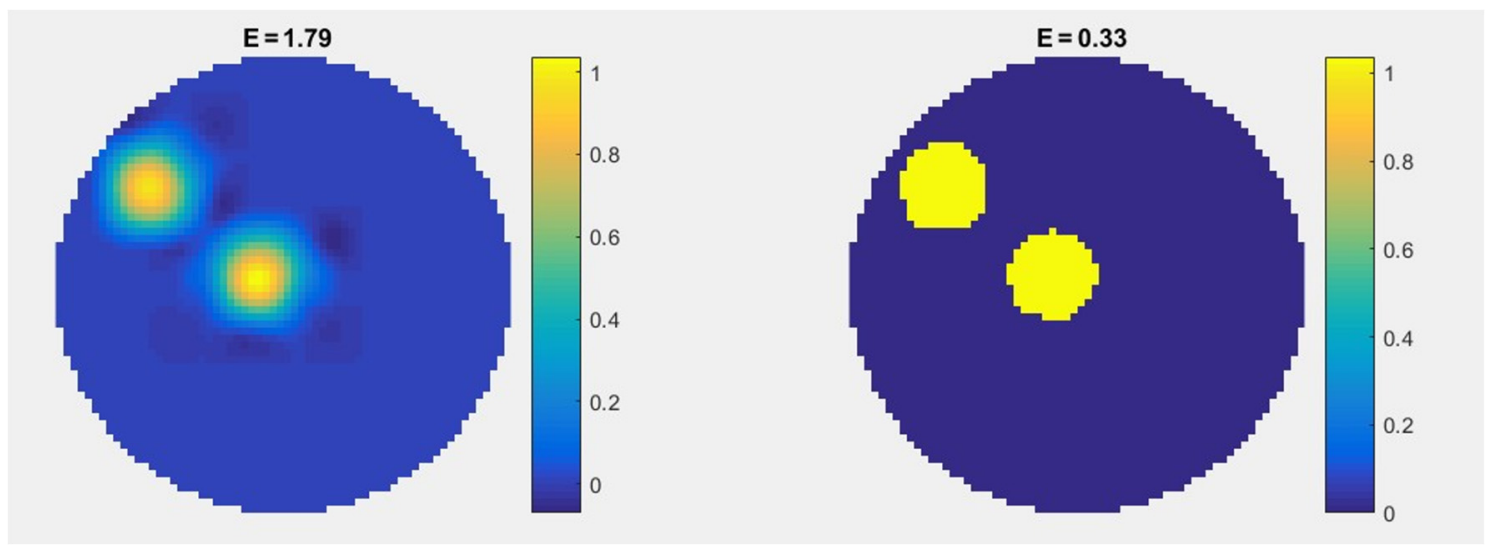

Figure 12. Filtering and image segmentation.

The picture achieved after filtering differs from the tomogram standard quality of other algorithms [31-35]. In addition, an exact image can be obtained after segmentation. The filtering gives satisfactorily high-quality results.

The last element worth checking is how the combination of compression and filtering can reproduce more complex shapes than simple ovals.

The results of experiments for more complex objects (Figure 13) are very positive. Despite the apparent loss of detail, the overall shape is reproduced correctly.

The only drawback is that narrow elements lose their fill uniformity after decompression due to the filtering method. Nevertheless, it is a basis for exploring other ways of filtering the images in future work. 

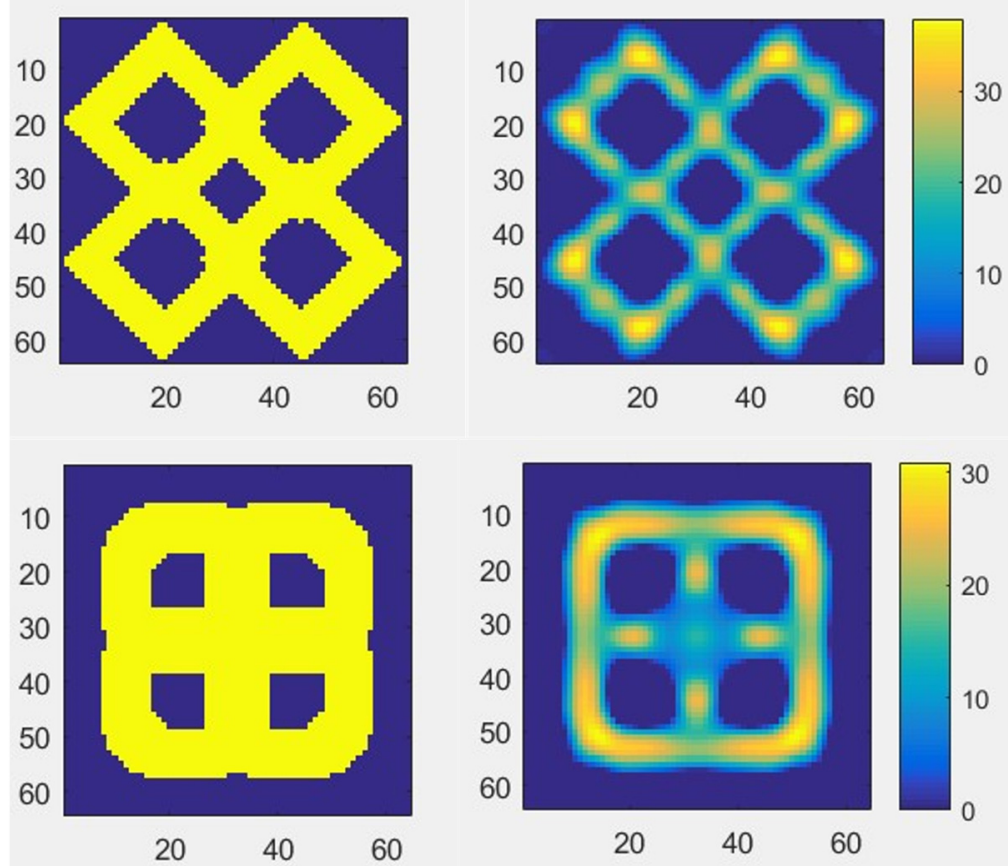

(a)
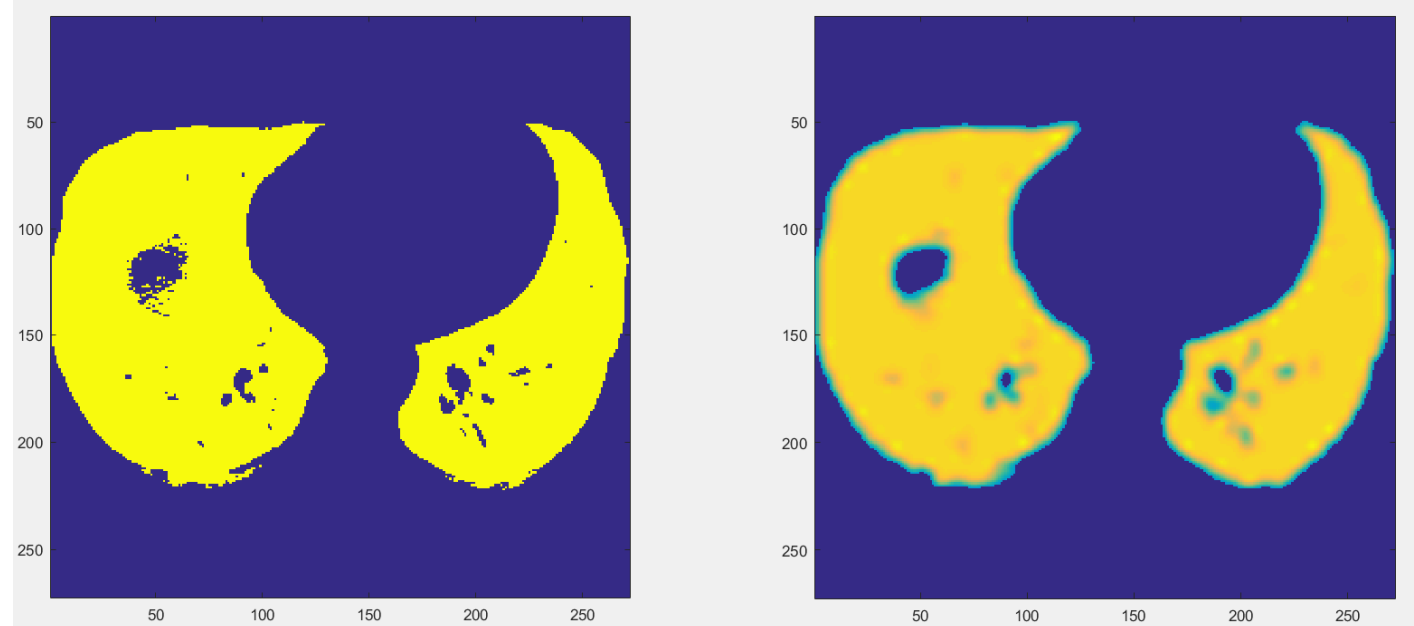

(b)

Figure 13. Examples of image reconstruction: (a) direct decompression with filtering, (b) reconstruction of a complex shape derived from computed tomography of the lung.

\subsubsection{Mathematical Model}

Previous studies conducted by different teams on the simple problem in ultrasonic transmission tomography have shown the effectiveness of methods based on radial models $[7,23,32]$.

Techniques for determining the sensitivity of a tomographic system along rays may vary depending on the method adopted; however, regardless of the method adopted, all ray models of the simple problem can be reduced to matrix form:

$$
m=J r
$$

where $r$-vector encoding the image values (reconstruction), 
$m$-vector encoding the measurement values,

$J$-matrix whose rows are obtained from individual sensitivity maps (determined by radial methods).

For this study, the smoothed thick lines model [32] was used as a starting point. The application of the selected sensitivity map is shown in Figure 14.
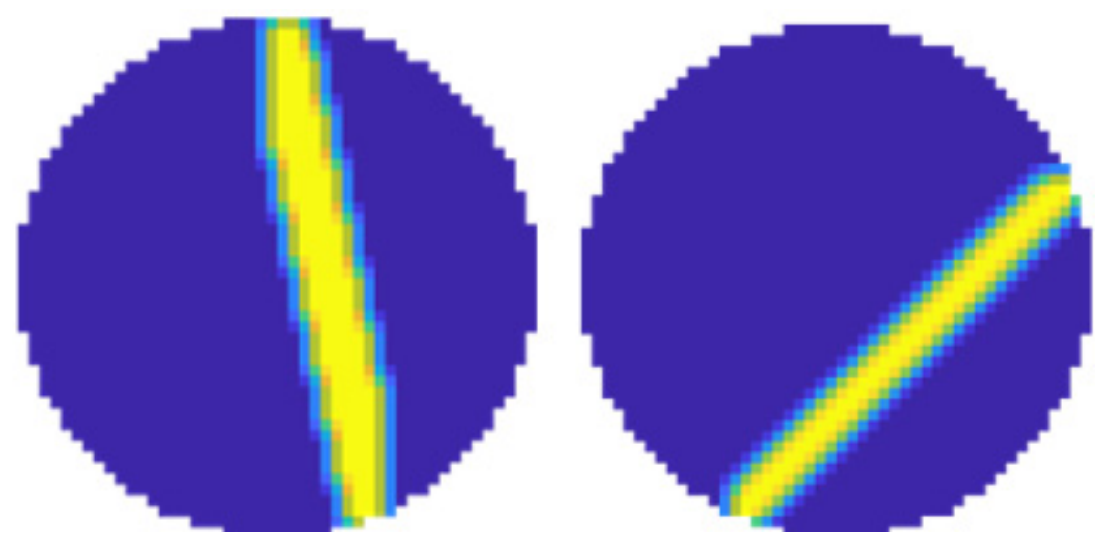

Figure 14. Example sensitivity maps.

It is extremely important to analyse the process of solving the inverse problem. This section presents how to transform the computational model so that compression is a uniform image reconstruction.

It is worth noting that the BWTR method can be applied to any model of the simple problem expressed by Equation (5). Thus, starting from the formulation of the simple problem for 16 sensors and a $48 \times 48$ pixel resolution, we have model (5), where $m \in \mathbb{R}^{120 \times 1}, J \in \mathbb{R}^{120 \times 2304}, r \in \mathbb{R}^{2304 \times 1}$. If one divides a $48 \times 48$ pixel image into $8 \times 8$ pixel blocks, it can be seen that, according to Equation (5), the measurement vector can be expressed as a superposition of the individual influences from each $8 \times 8$ pixel block:

$$
m=\sum_{i=1}^{6} \sum_{j=1}^{6} J^{i j} r_{i j}
$$

where $J^{i j} \in \mathbb{R}^{M \times 48}$ is a minor matrix of $J$, constructed from the columns of $J$ corresponding to the pixels in block indexed with $i, j$;

$r_{i j}$ is the part of vector $r$, which corresponds to the pixels of block $i, j$.

If we apply the compression to the $r_{i j}$ vector-based on the three basis vectors, then we obtain the projection described by the equation:

$$
\widetilde{r}_{i j}=b_{i j}^{(1)} B_{1}+b_{i j}^{(2)} B_{2}+b_{i j}^{(3)} B_{3}
$$

where: $b_{i j}^{(k)}, k=1,2,3$ are the coefficients of the $r_{i j}$ a vector in compression base. Returning to the forward model equation, we obtain:

$$
m \cong \sum_{i=1}^{6} \sum_{j=1}^{6} b_{i j}^{(1)} \underbrace{I^{i j} B_{1}}_{\substack{J_{D C T_{1}}^{i j} \\[M \times 1]}}+b_{i j}^{(2)} \underbrace{I^{i j} B_{2}}_{\substack{J_{D C T_{2}}^{i j} \\[M \times 1]}}+b_{i j}^{(3)} \underbrace{I^{i j} B_{2}}_{\substack{J_{D C T_{3}}^{i j} \\[M \times 1]}}
$$


which can be expressed as:

$$
\begin{aligned}
& m \cong \sum_{i=1}^{N} \sum_{j=1}^{N} \underbrace{\left[\begin{array}{lll}
J_{D C T_{1}}^{i j} & J_{D C T_{2}}^{i j} & J_{D C T_{3}}^{i j}
\end{array}\right]}_{J^{i j}\left[\begin{array}{lll}
B_{1} & B_{2} & B_{3}
\end{array}\right]}\left[\begin{array}{c}
b_{i j}^{(1)} \\
b_{i j}^{(2)} \\
b_{i j}^{(3)}
\end{array}\right]_{[3 \times 1]} \\
& \text { [ } M \times 3]
\end{aligned}
$$

and further using the notation:

$$
J_{D C T}^{i j}=J^{i j}\left[\begin{array}{lll}
B_{1} & B_{2} & B_{3}
\end{array}\right], \quad \boldsymbol{b}_{i j}=\left[\begin{array}{c}
b_{i j}^{(1)} \\
b_{i j}^{(2)} \\
b_{i j}^{(3)}
\end{array}\right]
$$

and switching to single-index numbering

$$
m \cong \sum_{k=1}^{N^{2}} J_{D C T}^{k} \boldsymbol{b}_{k}
$$

we can see that this equation can be rewritten as:

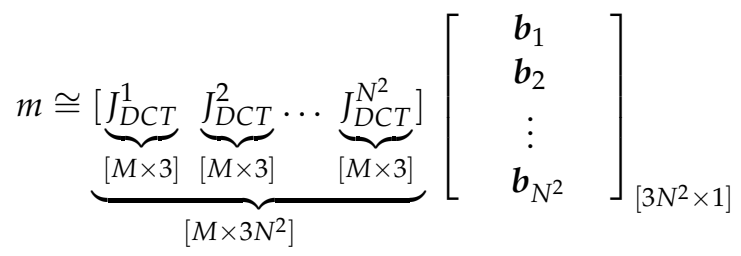

and thus, we can obtain a reconstruction model based on the coefficient vector in the new basis (i.e., a compressed model). We can reformulate it as follows:

$$
m \cong J_{D C T} \boldsymbol{b}
$$

For convenience, by changing the indexing method from 2-index to 1-index, the parts of Equation (13) can be represented as:

$$
J_{D C T}=\left[\begin{array}{lll}
J_{D C T}^{1} & J_{D C T}^{2} \cdots J_{D C T}^{6^{2}}
\end{array}\right], \boldsymbol{b}=\left[\begin{array}{c}
\boldsymbol{b}_{1} \\
\boldsymbol{b}_{2} \\
\vdots \\
\boldsymbol{b}_{6^{2}}
\end{array}\right]_{108}
$$

where:

$\boldsymbol{b}_{i}, i \in\left\{1 \ldots 6^{2}\right\}$ are column vectors for the coefficients of the DCT transform in the block with index $i$;

$J_{D C T}^{i}$ represents the transformation of the matrix $J^{i j}$ from a canonical base into a DCT.

Having a model, the vector $b$ can be reconstructed. The compressed image is the result of the direct representation of the reconstructed vector. Because we know from Hilbert space theory that two spaces of equal dimensionality are isometric concerning $l_{2}$ norms of coordinates, the proper inverse operator can be obtained by minimising the second norm in the transform space:

$$
\boldsymbol{b}_{\text {rec }}=J_{D C T}^{i n v} m
$$

Thus, $J_{D C T}^{i n v}$ is a matrix expressed by the formula:

$$
J_{D C T}^{i n v}=\left(J_{D C T}^{\mathrm{T}} J_{D C T}+\lambda \mathrm{I}\right)^{-1} J_{D C T}^{T}
$$


It is important to note that in connection with the transition to an overdetermined system, regularisation of the solution by adding the $\lambda I$ term is only necessary due to the poor conditioning of the $J_{D C T}$ but is not required due to the ambiguity of the mean square solution.

Thus, the scalar $\lambda$ is chosen only to minimise the effect of numerical errors in matrix inversion, and thus the process of selecting the parameter $\lambda$ is simplified and can be based on the observation that the conditioning of the $J_{D C T}^{\mathbf{T}} J_{D C T}+\lambda \mathrm{I}$ is easily expressed as a function of $\lambda$ :

$$
\operatorname{cond}\left(J_{D C T}^{\mathbf{T}} J_{D C T}+\lambda I\right)=C(\lambda)=\frac{\lambda_{\max }+\lambda}{\lambda_{\min }+\lambda}
$$

where $\lambda_{\max }, \lambda_{\min }$ are the greatest and the smallest eigenvalues of $J_{D C T}^{\mathbf{T}} J_{D C T}$.

Thus, one can easily see that setting the regularisation parameter to $\lambda=100$ or higher should be sufficient to obtain a satisfactory result. The conditioning of the equation as a function of the regularisation parameter is shown in Figure 15.

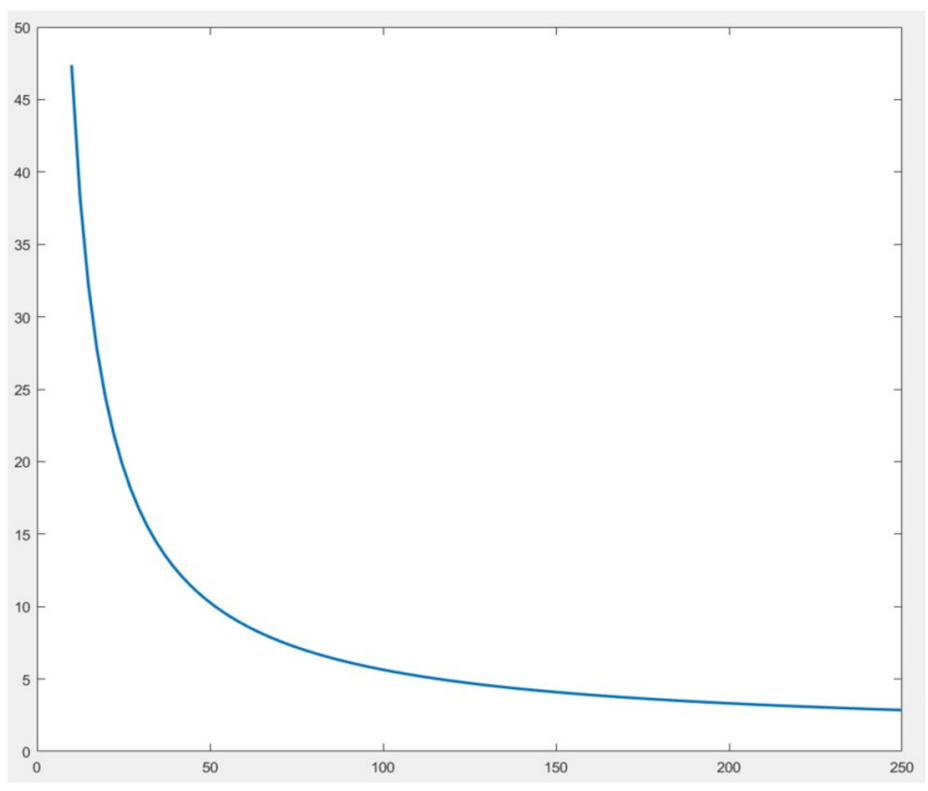

Figure 15. Conditioning of the equation as a function of the regularisation parameter.

\subsection{Innovation of the Solution}

The novelties in this paper are both the apparatus (sensors, computer systems) and the algorithm used to solve the inverse problem. Given the frequency and number of novel research developments for innovative process solutions in the industry, UST is less advanced than other tomographic methods. It could be because of the difficulty in developing an effective system incorporating the sensors and algorithms to enable precise image reconstruction in a medical setting. The traditional approach to modelling an ultrasonic tomograph is to approximate the system behaviour with a model of simple propagation rays between two ultrasonic probes. Publications verify the effectiveness of this solution reliably being able to image the positions of inclusions and the domain of the tomographic system [27-35]. Moreover, the effectiveness of such models can be verified by a practical study from measurement data. Despite these facts, it is still the case that there is no ideal physical model of UST, so a better understanding of this domain is desirable.

The presented method uses lossy image compression to reconstruct the number of unknowns by dividing the image into square sides. By applying a transform, we enable compression on each block. This paper uses DCT for compression, but it is worth emphasizing that this method is not restricted to this particular orthonormal base. 


\section{Results}

Specific tests using ultrasonic transmission tomography were performed, which confirm the correctness of the proposed method. The tank, which was made of acrylic glass, was used for the experiments. The reservoir with the placed air artefacts was filled with water. They were made of PVC pipe and sealed on one side. The results of the tests are shown in Figure 16, which shows single and double inclusions and reconstruction combined with segmentation.
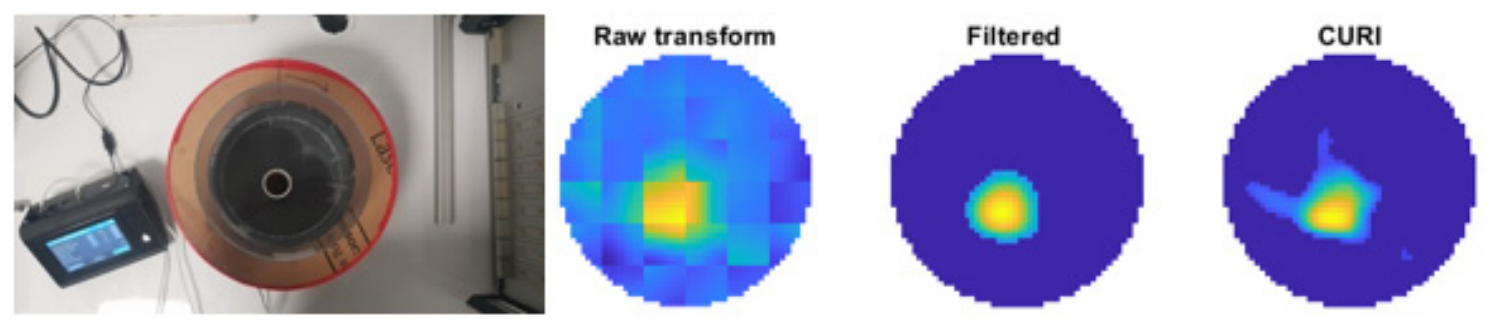

(a)
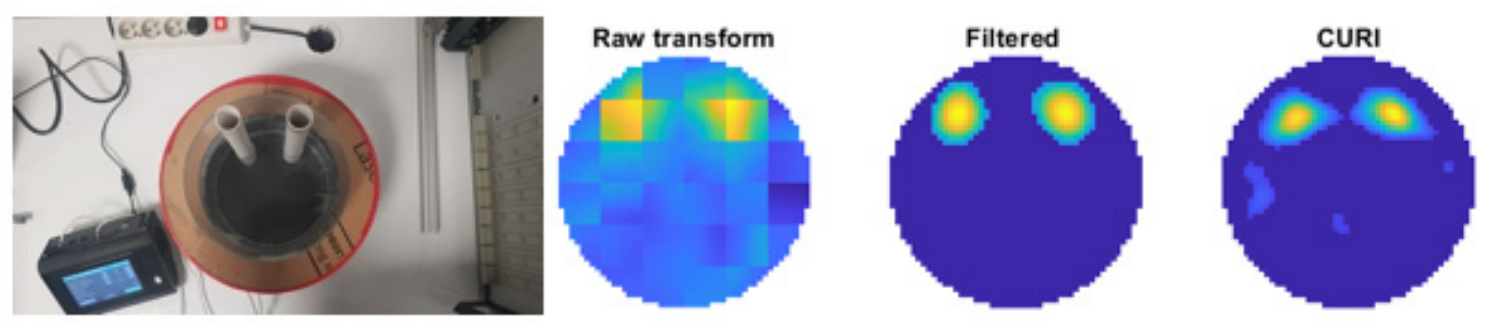

(b)
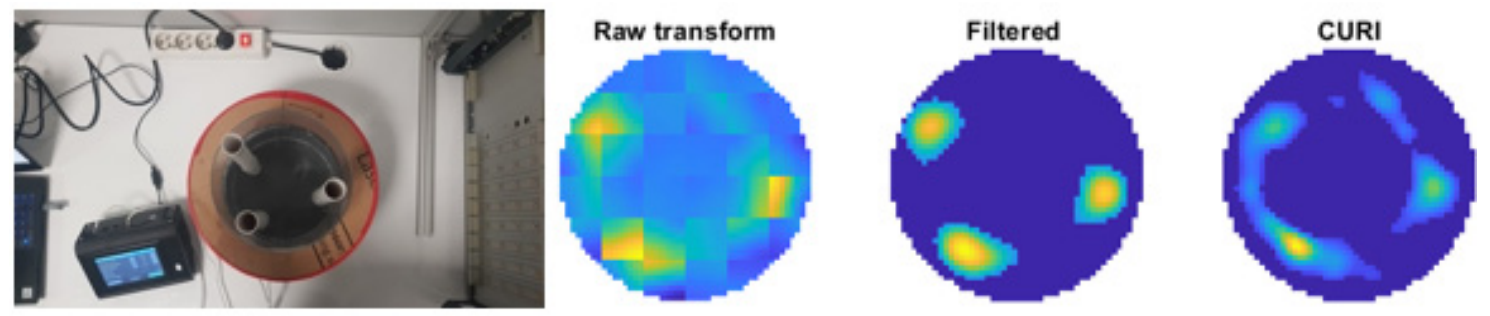

(c)
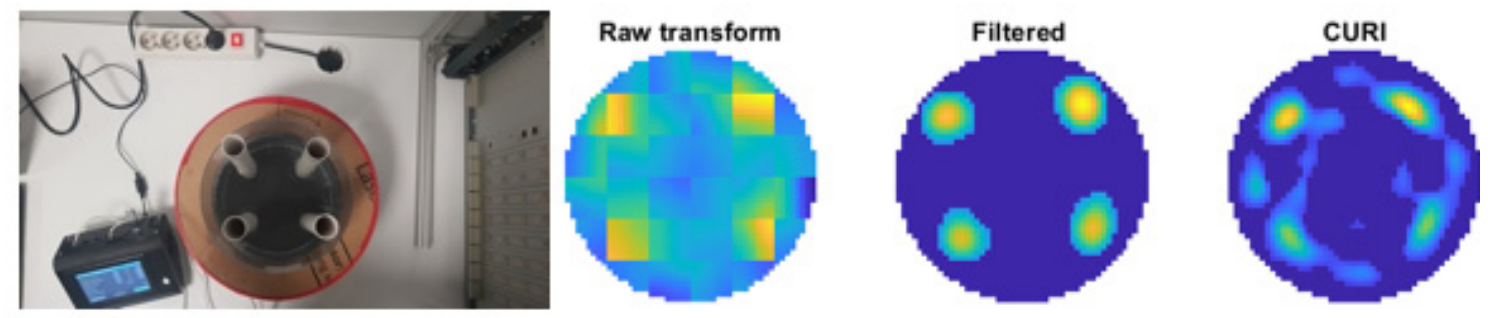

(d)

Figure 16. Measurement system and examples of the image reconstruction for (a) 1 object, (b) 2 objects, (c) 3 objects, and (d) 4 objects using UST. 
The first reconstruction on the left is without filtering. The result of the algorithm with filtering and thresholding is shown in the next figure. The last one shows the reconstruction obtained presented in our previous work [31,32,35].

Presented image reconstructions are of good quality in spite of performing reduction of the system of equations. Therefore, it can be seen that the results are stabilising, and there is an improvement in quality and a reduction in artefacts, which means that the method is robust to measurement noise [35]. It also saves memory by archiving the results directly into compressed form, where no server-side compression is required. Furthermore, it enables the archived result to be obtained in less time due to reduced systems of formulas.

\section{Comparison of Image Reconstructions}

Three well-known indicators were used to assess the quality of the reconstruction from the BWTR method: relative image error (RIE), mean square error (MSE), and image correlation coefficient (ICC). The MSE metric was evaluated according to the equation:

$$
\operatorname{MSE}=\frac{1}{n} \sum_{i=1}^{n}\left(y_{i}^{\prime}-y_{i}^{*}\right)^{2}
$$

where: $n$-number of pixels in the image, $y_{i}^{\prime}$-reference of $i$ th pixel, and $y^{*}{ }_{i}$-value of $i$ th reconstructed pixel.

The next measure of reconstruction quality RIE was calculated according to the equation:

$$
R I E=\frac{\left\|y^{\prime}-y^{*}\right\|}{\left\|y^{\prime}\right\|}
$$

The quality ICC was calculated according to the equation:

$$
I C C=\frac{\sum_{i=1}^{n}\left(y_{i}^{*}-\bar{y}^{*}\right)\left(y_{i}^{\prime}-\bar{y}^{\prime}\right)}{\sqrt{\sum_{i=1}^{n}\left(y_{i}^{*}-\bar{y}^{*}\right)^{2} \sum_{i=1}^{n}\left(y_{i}^{\prime}-\bar{y}^{\prime}\right)^{2}}}
$$

where $\bar{y}^{\prime}$ is the mean value for reference pixels and $\bar{y}^{*}$ is the mean value for reconstructed pixels. ICC is used to determine the correlation between the reference image and the reconstruction image based on Pearson's correlation coefficient. If the ICC is closer to 1, the correlation of the output image to the reference image is better, resulting in a more accurate reconstruction. Image quality is better the smaller the values of the MSE and RIE metrics.

Table 1 shows the reconstruction quality analysis of the reconstructed images using the BWTR method. The corresponding column headings present data on the number of hidden objects $(\mathrm{O} 1, \mathrm{O} 2, \mathrm{O} 3, \mathrm{O} 4)$.

Table 1. Image reconstruction indicators-BWTR method.

\begin{tabular}{cccccc}
\hline \multirow{2}{*}{ Method of Reconstruction } & \multirow{2}{*}{ Indicator } & \multicolumn{4}{c}{ Variant } \\
\cline { 3 - 6 } & & $\mathbf{O 1}$ & $\mathbf{O 2}$ & $\mathbf{O 3}$ & $\mathbf{O 4}$ \\
\hline \multirow{3}{*}{ BTWR } & MSE & 0.0087 & 0.0156 & 0.0300 & 0.0466 \\
& RIE & 0.2538 & 0.2340 & 0.2962 & 0.3618 \\
& ICC & 0.8588 & 0.8873 & 0.8506 & 0.8131 \\
& Time of reconstruction [s] & \multicolumn{4}{c}{0.0014896} \\
\hline
\end{tabular}

BWTR has been demonstrated for efficient compression and decompression of typical tomograms, allowing testing on real measurements. The BWTR method changes the canonical base to an orthonormal base and encodes the image content instead of individual pixels.

For comparison, the values of the quantitative coefficients for the Corrected Uniform Ray Integration (CURI) method were added in Table 2 due to the fact that the visual 
evaluation of the reconstruction is not precise and insufficient to compare the two methods analyzed objectively.

Table 2. Image reconstruction indicators-CURI method.

\begin{tabular}{cccccc}
\hline \multirow{2}{*}{$\begin{array}{c}\text { Method of } \\
\text { Reconstruction }\end{array}$} & Indicator & \multicolumn{4}{c}{ Variant } \\
\cline { 3 - 6 } & & O1 & O2 & O3 & O4 \\
\hline \multirow{2}{*}{ CURI } & MSE & 0.0105 & 0.0266 & 0.0554 & 0.0694 \\
& RIE & 0.3066 & 0.3984 & 0.5482 & 0.5385 \\
& ICC & 0.8285 & 0.7873 & 0.6788 & 0.6562 \\
\hline
\end{tabular}

\section{Conclusions}

This paper presents an innovative idea of an open architecture sensor platform for visualising processes in the industry. The method is presented in various aspects, considering the hardware and algorithmic layers as a cyber-physical system with a cloud computing mechanism for data acquisition, collection, and analysis.

The reconstruction method implemented in transmission tomography is modelled on the block-wise-transform-reduction. For this purpose, a measuring device was designed together with an original algorithm of image reconstruction.

The research has shown that UST effectively analyses test objects in closed tanks and allows process analysis to be performed inside the test object without interfering with it. The presented technique works well in practice and has some important benefits. First, it enables rapid, real-time visualization. The result is received very quickly in compressed form. Second, the algorithm is amenable to modification for particular implementations. This makes it possible to change the fundamentals within selective imaging. It also allows sensor information to be used directly, allowing more measurements to be taken, thus increasing the number of basis vectors. This arrangement expands the range of shapes in the compression subspace and enables a faithful representation of the shapes in the image. The individual results show that the compression process produces more robust reconstruction results. A thorough understanding of the precise nature of this type of phenomenon requires continued research work. Considering the Internet of Things, distributed systems and computer networks, direct compression in reconstruction methods reduces the amount of data sent between network elements.

Fast acquisition of measurement data in combination with fast and accurate image reconstruction algorithms allows for precise spatial determination of material parameters necessary to control the mixing phases of liquid and gaseous substances. Tomographic technologies can be used offline to monitor the performance of independent devices or for online measurement, control, and analysis of industrial processes. Therefore, it can be used to monitor mixing, separation, flow, and reactor dynamics in both single and multiphase processes. The obtained data can monitor process responses, improve product quality and performance, monitor flow concentrations, and provide data for process control.

Future work will address improvements to the block-wise-transform-reduction algorithm in deep learning image reconstruction and ultrasound tomography measurement devices.

Author Contributions: Development of the concept of algorithms and implementation of the image reconstruction in ultrasound transmission tomography, K.K.; development of the system concept, measurement methodology, image reconstruction, and supervision, T.R.; preparation of measurements, development of research methodology, preparation of measurement documentation, and development numerical methods and techniques, P.O., S.S.-A. and M.G.; preparation of research methodology, literature review, formal analysis, general review, and editing of the manuscript, M.M. All authors have read and agreed to the published version of the manuscript.

Funding: This research received no external funding.

Institutional Review Board Statement: Not applicable. 
Informed Consent Statement: Not applicable.

Data Availability Statement: Not applicable.

Conflicts of Interest: The authors declare no conflict of interest.

\section{References}

1. Bergweiler, S. Intelligent Manufacturing based on Self-Monitoring Cyber-Physical Systems. In Proceedings of the Ninth International Conference on Mobile Ubiquitous Computing, Systems, Services and Technologies (UBICOMM 2015), Nice, France, 19-24 July 2015; pp. 108-113.

2. Wan, J.; Tang, S.; Shu, Z.; Li, D.; Wang, S.; Imran, M.; Vasilakos, A.V. Software-Defined Industrial Internet of Things in the Context of Industry 4.0. IEEE Sens. J. 2016, 16, 7373-7380. [CrossRef]

3. Wang, S.; Wan, J.; Li, D.; Zhang, C. Implementing Smart Factory of Industrie 4.0: An Outlook. Int. J. Distrib. Sens. Netw. 2016, 12, 3159805. [CrossRef]

4. Herman, G.T. Image Reconstruction from Projections: The Fundamentals of Computerised Tomography; Academic Press: New York, NY, USA, 1980.

5. Polakowski, K.; Sikora, J. Podstawy Matematyczne Obrazowania Ultradźwiękowego; Politechnika Lubelska: Lublin, Poland, 2016. (In Polish)

6. Kak, A.C.; Slaney, M. Principles of Computerized Tomographic Imaging; IEEE Press: New York, NY, USA, 1999.

7. Koulountzios, P.; Rymarczyk, T.; Soleimani, M. Ultrasonic Time-of-Flight Computed Tomography for Investigation of Batch Crystallisation Processes. Sensors 2021, 21, 639. [CrossRef]

8. Mosorov, V.; Grudzień, K.; Sankowski, D. Flow velocity measurement methods using electrical capacitance tomography. Inform. Autom. Pomiary Gospod. Ochr. Śr. (IAPGOŚ) 2017, 7, 30-36. [CrossRef]

9. Kłosowski, G.; Rymarczyk, T.; Kania, K.; Świć, A.; Cieplak, T. Maintenance of industrial reactors based on deep learning driven ultrasound tomography. Eksploat. Niezawodn. Maint. Reliab. 2020, 22, 138-147. [CrossRef]

10. Koulountzios, P.; Rymarczyk, T.; Soleimani, M. A quantitative ultrasonic travel-time tomography system for investigation of liquid compounds elaborations in industrial processes. Sensors 2019, 19, 5117. [CrossRef]

11. Beck, M.S.; Williams, R.A. Process tomography: A European innovation and its applications. Meas. Sci. Technol. 1996, 7, 215-224. [CrossRef]

12. Majchrowicz, M.; Kapusta, P.; Jackowska-Strumiłło, L.; Sankowski, D. Acceleration of image reconstruction process in the electrical capacitance tomography 3D in heterogeneous, multi-GPU system. Inform. Control Meas. Econ. Environ. Prot. 2017, 7, 37-41. [CrossRef]

13. Banasiak, R.; Wajman, R.; Jaworski, T.; Fiderek, P.; Fidos, H.; Nowakowski, J.; Sankowski, D. Study on two-phase flow regime visualisation and identification using $3 \mathrm{D}$ electrical capacitance tomography and fuzzy-logic classification. Int. J. Multiph. Flow 2014, 58, 1-14. [CrossRef]

14. Rybak, G.; Strzecha, K. Short-Time Fourier Transform Based on Metaprogramming and the Stockham Optimization Method. Sensors 2021, 21, 4123. [CrossRef] [PubMed]

15. Dusek, J.; Mikulka, J. Measurement-Based Domain Parameter Optimisation in Electrical Impedance Tomography Imaging. Sensors 2021, 21, 2507. [CrossRef] [PubMed]

16. Kozłowski, E.; Mazurkiewicz, D.; Żabiński, T.; Prucnal, S.; Sęp, J. Assessment model of cutting tool condition for real-time supervision system. Eksploat. Niezawodn. Maint. Reliab. 2019, 21, 679-685. [CrossRef]

17. Duraj, A.; Korzeniewska, E.; Krawczyk, A. Classification algorithms to identify changes in resistance. Prz. Elektrotech. 2015, 1, 82-84. [CrossRef]

18. Krawczyk, A.; Korzeniewska, E. Magnetophosphenes-history and contemporary implications. Prz. Elektrotech. 2018, 1, 63-66. [CrossRef]

19. Du, X.; Li, J.; Feng, H.; Chen, S. Image Reconstruction of Internal Defects in Wood Based on Segmented Propagation Rays of StressWaves. Appl. Sci. 2018, 8, 1778. [CrossRef]

20. Rymarczyk, T.; Kłosowski, G. Innovative methods of neural reconstruction for tomographic images in maintenance of tank industrial reactors. Eksploat. Niezawodn. Maint. Reliab. 2019, 21, 261-267. [CrossRef]

21. Rymarczyk, T. Characterization of the shape of unknown objects by inverse numerical methods. Prz. Elektrotech. 2012, 88, 138-140.

22. Mazurek, M.; Rymarczyk, T.; Kłosowski, G.; Maj, M.; Adamkiewicz, P. Tomographic measuring sensors system for analysis and visualisation of technological processes. In Proceedings of the 50th Annual IEEE-IFIP International Conference on Dependable Systems and Networks-Supplemental Volume (DSN-S), Valencia, Spain, 29 June-2 July 2020; pp. 45-46.

23. Cheng, X.; Li, Q.; Zhou, Z.; Luo, Z.; Liu, M.; Liu, L. Research on a Seepage Monitoring Model of a High Core Rockfill Dam Based on Machine Learning. Sensors 2018, 18, 2749. [CrossRef]

24. Soetomo, K.; Rahma, T.F.; Juliastuti, E.; Kurniadi, D. Ultrasonic tomography for reinforced concrete inspection using algebraic reconstruction technique with Iterative Kaczmarz method. In Proceedings of the 2016 International Conference on Instrumentation, Control and Automation (ICA), Bandung, Indonesia, 29-31 August 2016; IEEE: Piscataway, NJ, USA, 2016 ; pp. 16-21. 
25. Yan, B.; Wu, C.; Ma, H. Study on the method of nonmetallic defects based on ultrasonic tomography and morphology. In Proceedings of the 2017 12th IEEE Conference on Industrial Electronics and Applications (ICIEA), Siem Reap, Cambodia, 18-20 June 2017; IEEE: Piscataway, NJ, USA, 2017; pp. 1287-1292.

26. Mohd Yunus, F.R.; Noor Azlan, N.A.; Nor Ayob, N.M.; Pusppanathan, M.J.; Jumaah, M.F.; Goh, C.L.; Abdul Rahim, R.; Ahmad, A.; Yunus, Y.M.; Abdul Rahim, H. Simulation Study of Bubble Detection Using Dual-Mode Electrical Resistance and Ultrasonic Transmission Tomography for Two-Phase Liquid and Gas. Sens. Transducers 2013, 150, 97-105.

27. Koulountzios, P.; Rymarczyk, T.; Soleimani, M. A triple-modality ultrasound computed tomography based on full-waveform data for industrial processes. IEEE Sens. J. 2021, 21, 20896-20909. [CrossRef]

28. Rymarczyk, T.; Kłosowski, G. The use of elastic net and neural networks in industrial process tomography. Prz. Elektrotech. 2019, 95, 59-62. [CrossRef]

29. Rymarczyk, T.; Kozłowski, E.; Kłosowski, G. Electrical impedance tomography in 3D flood embankments testing-Elastic net approach. Trans. Inst. Meas. Control 2019, 42, 680-690. [CrossRef]

30. Szumowski, J.; Oleszek, M.; Rymarczyk, T.; Adamkiewicz, P.; Sikora, J. Hybrid tomographic device for acquisition of electrical tomography data. In Proceedings of the 2018 International Interdisciplinary PhD Workshop (IIPhDW 2018), Swinoujscie, Poland, 9-12 May 2018; pp. 162-164.

31. Mazurek, M.; Kania, K.; Rymarczyk, T.; Wójcik, D.; Cieplak, T.; Gołąbek, P. Implementation of Block-Wise-Transform-Reduction Method for Image Reconstruction in Ultrasound Transmission Tomography. In Proceedings of the INFOCOM 2021 IEEE Conference on Computer Communications, Virtual Conference, 10-13 May 2021.

32. Mazurek, M.; Rymarczyk, T.; Kania, K.; Kłosowski, G. Dedicated algorithm based on discrete cosine transform for the analysis of industrial processes using ultrasound tomography. In Adjunct Proceedings of the 2020 ACM International Joint Conference on Pervasive and Ubiquitous Computing and Proceedings of the 2020 ACM International Symposium on Wearable Computers, Proceedings of the 2020 ACM International Joint Conference on Pervasive and Ubiquitous Computing and Proceedings of the 2020 ACM International Symposium on Wearable Computers, Virtual Event Mexico, 12-17 September 2020; The Association for Computing Machinery: New York, NY, USA, 2020.

33. Kania, K.; Rymarczyk, T.; Maj, M.; Golabek, M. Implementation of Fermat's Principle for Detection of Anomalies in Ultrasound Transmission Tomography. Appl. Electromagn. Mod. Eng. Med. PTZE 2019, 2019, 67-71.

34. Kania, K.; Maj, M.; Rymarczyk, T.; Adamkieicz, P.; Golabek, M. Image reconstruction in ultrasound transmission tomography using the Fermat's Principle. Prz. Elektrotech. 2020, 96, 186-189. [CrossRef]

35. Kania, K.; Rymarczyk, T.; Maj, M.; Gołąbek, M.; Adamkiewicz, P.; Sikora, J. RayIntegration methods for real-time reconstruction using a compact measuring device. In Proceedings of the 2019 International Interdisciplinary PhD Workshop (IIPhDW), Wismar, Germany, 15-17 May 2019; pp. 47-50. [CrossRef] 